\title{
Thrombotic thrombocytopenic purpura-hemolytic uremic syndrome in pregnancy in a known case of antiphospholipid antibody syndrome
}

\author{
Nitin Pai Dhungat, Shubhdeep Kaur*
}

Department of Obstetrics and Gynaecology, Bombay Hospital Institute of Medical Sciences, Mumbai, Maharashtra, India

Received: 06 January 2022

Revised: 03 February 2022

Accepted: 11 February 2022

\section{*Correspondence:}

Dr. Shubhdeep Kaur,

E-mail: shubhdeepkaurdadiala@gmail.com

Copyright: (c) the author(s), publisher and licensee Medip Academy. This is an open-access article distributed under the terms of the Creative Commons Attribution Non-Commercial License, which permits unrestricted non-commercial use, distribution, and reproduction in any medium, provided the original work is properly cited.

\begin{abstract}
Thrombotic thrombocytopenic purpura is a haematological disorder which may be severely life-threatening. It affects the microcirculation of multiple organ systems. One of the precipitating factors for thrombotic thrombocytopenic purpura is pregnancy. The case report describes a 32 years old patient who presented with antepartum TTP-HUS and was initially treated as a case of HELLP syndrome without any improvement. The introduction of aggressive treatment with plasma transfusion and plasmapheresis has improved maternal and fetal survival rates in these cases. thrombotic thrombocytopenic purpura-haemolytic uremic syndrome is a rare complication of pregnancy and parturition, that may simulate the more common obstetric complications, namely, preeclampsia and the syndrome of haemolysis, elevated liver functions tests, low platelets. Review of the previously reported cases of pregnancy-related TTP and the current treatment options for this rare condition are also discussed. Careful diagnosis, monitoring, and treatment in congenital and acquired TTP have assisted in excellent pregnancy outcomes.
\end{abstract}

Keywords: Thrombotic thrombocytopenic purpura, HELLP syndrome, Haemolytic uremic syndrome, Plasma exchange transfusion

\section{INTRODUCTION}

Thrombotic thrombocytopenic purpura-haemolytic uremic syndrome (TTP-HUS) is an infrequent occlusive microangiopathic disorder that is caused by intravascular platelet aggregation. There is a severe deficiency of ADAMTS-13, a metalloprotease which functions by cleaving ultra large multimers of von Willebrand factor (vWF). The resultant large von Willebrand factor multimers increase platelet adhesiveness and impair fibrinolytic activity, with subsequent thrombotic occlusion of the microvasculature. ${ }^{1}$ For uncertain reasons, TTP-HUS may occur or may be precipitated in late pregnancy or in the postpartum period. One of the causes is pregnancy causing physiological decrease in the levels of ADAMTS-13, though their levels still remain about 5-
$10 \%$ which is coherent with TTP- HUS. There is a classical pentad of microangiopathic haemolytic anaemia, thrombocytopenia, fluctuating central nervous system abnormalities, fever, and renal impairment occurs in only about $6-40 \%$ of patients. ${ }^{2-4}$

However, $75 \%$ of the patients may present with the triad of anaemia, thrombocytopenia, bizarre neurological abnormalities. Non-haematological findings such as fever, renal symptoms, neurological signs may not be consistent in all patients hence, the diagnosis of TTPHUS is often made by a dyad of thrombocytopenia and micro angio-pathic haemolytic anaemia. ${ }^{3}$ HELLP syndrome has a very similar presentation. An accurate diagnosis is therefore essential if these syndromes have to be managed properly. 


\section{CASE REPORT}

A 32 year old female, G4 P0 A3, by date 31 weeks, by scan corresponding with the dates, was brought to the casualty in a drowsy state due to a seizure, referred from a primary care centre. On examination her heart rate was $68 \mathrm{bpm}$, blood pressure was 160/100 mm hg, capillary prick sugar was $92 \mathrm{mg} / \mathrm{dl}$. She was catheterised in the primary care centre, and the urine reservoir bag contained $100 \mathrm{mls}$ of cola-coloured urine. Dipstick urine albumin was +1 . Upon per abdomen examination, uterus corresponded to 30-32 weeks, with an unstable lie, relaxed, foetal heart rate 150 beats per minute and regular. The blood reports from the referral centre showed thrombocytopenia and anaemia. She had received 1 pint of packed cell volume and 4 random donor platelets before her referral. She was admitted in the ICU and was given all preliminary treatment, including antiseizure medication. An intensivist, a neurologist, and a haematologist were consulted, who suspected APLA with bicytopenia. Within 12 hours of her ICU stay, patient had fall in her urine output and hence, a nephrologist was also consulted.

\section{Lab investigations sent immediately on admission}

Routine blood investigations- Complete blood count, manual platelet count, Liver function test, renal function test, random blood sugars, complete coagulation profile, LDH, peripheral smear for schistocytes, urine routine microscopy. 2D Echo was done due to raised blood pressure and it was within normal limits. MR angiogram of brain was done for seizure history and showed evidence of encephalitis of viral or autoimmune aetiology. Ultra sound with doppler study was done. It showed a single, live, intrauterine pregnancy of 32 weeks, cephalic presentation, amniotic fluid index (AFI)12 centimetres, a fundal placenta and expected foetal weight (EFW) $1210 \mathrm{~g}$.

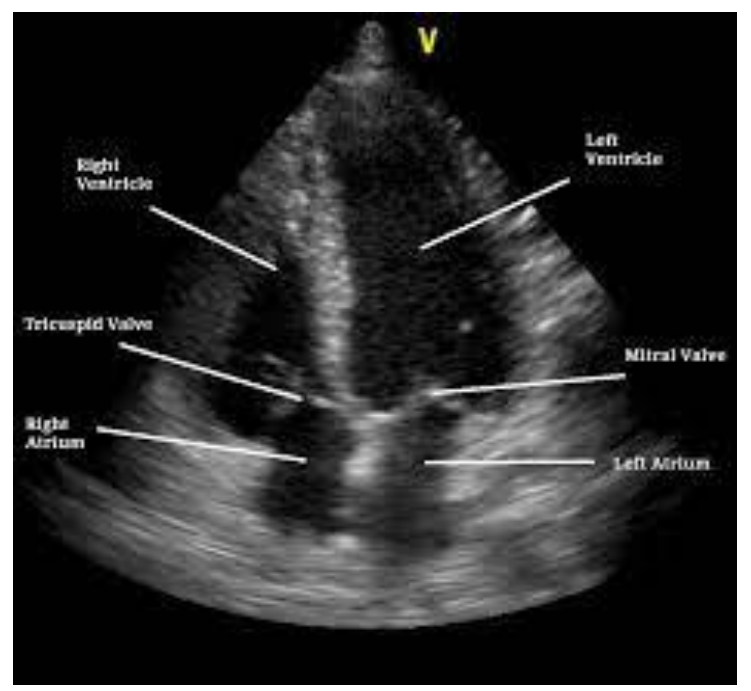

Figure 1: 2D echo investigation immediately on admission.

\section{Lab reports on admission}

Patient had haemoglobin <6gm \%, platelets $11000 / \mathrm{mm} 3$, liver enzymes were deranged, renal function tests were deranged, coagulation profile was near normal, lactate dehydrogenase was $>2000$, urine routine microscopy had >150-200 red blood cells/hpf, peripheral smear for schistocytes $>5 \%$, low haptoglobin, increased indirect bilirubin, direct coombs test was negative, platelet counts weren't improving despite the platelet transfusions hence ADAMTS - 13 study was done which showed: ADAMTS activity of $13 \%$ (normal range 50 to $150 \%$ ) ADAMTS autoantibody assay $2.8 \mathrm{AU} / \mathrm{ml}$ (Normal range <10\%)

\section{Plan of action}

Decision was taken to start heparin free plasma exchange for the patient with fresh frozen plasma. Packed cell volume was transfused depending on her daily haemoglobin values. After 3 cycles of plasma exchange without any improvement in the platelet count, a decision to do lower segment caesarean section was taken in the hopes that a termination of pregnancy would improve the patient's condition. Adequate measures were taken for the need of massive blood transfusions, platelet transfusion and fluid transfusion intraoperatively. Adequate measures were taken for neonatal resuscitation.

\section{Intraoperative findings}

At platelet count of 60,000 , Lower segment caesarean section was done under GA. Intraoperatively 1 pint platelets and 1 packed cells were transfused. Patient was kept on elective ventilatory support. A healthy female baby of 1210 grams was delivered and shifted to NICU for observation. The baby did not develop any respiratory complications, and was not found to have any obvious congenital anomalies. The patient was shifted back to the ICU after the caesarean section.

\section{Clinical course of the patient}

Day 2: injection of a low molecular weight heparin was started. Compression stockings were used as DVT prophylaxis. Day 3: Trial of weaning off ventilator failed. Platelet count was $80000 / \mathrm{mm} 3$ and hence, lumbar puncture could be done safely and it was done, to rule out viral/bacterial cause for encephalitis. Day 4: Fever of 101 Fahrenheit was recorded and fever profile, blood BACTEC were done. Jugular lines were changed from right to left side and the tips of these lines were sent for culture sensitivity; growth of Klebsiella, sensitive to meropenem, teicoplanin was seen. endotracheal culture showed growth of pseudomonas. Day 5: Fall of urine output occurred, with creatinine values of 4.2. The nephrologist diagnosed AKI and recommended haemodialysis, along with swapping low molecular weight heparin with dalteparin. Day 6: Patient had a circulatory collapse with bradycardia. An emergency 2D Echo showed LVEF of $20 \%$ with global hypokinesia, 
which were suspicious of myocarditis. A cardiologist was consulted who diagnosed it as stress induced cardiomyopathy and advised to correct the electrolyte imbalance. Since a bacterial/viral cause of encephalitis was ruled out, the haematologist had started rituximab and mycophenolate mofetil with methylprednisolone. Day 7: Patient had tachycardia with desaturation episode. An emergency chest ultrasound showed bilateral pleural effusion with lower lobe consolidation. Lasix infusion was started and USG guided pleural tap was done.
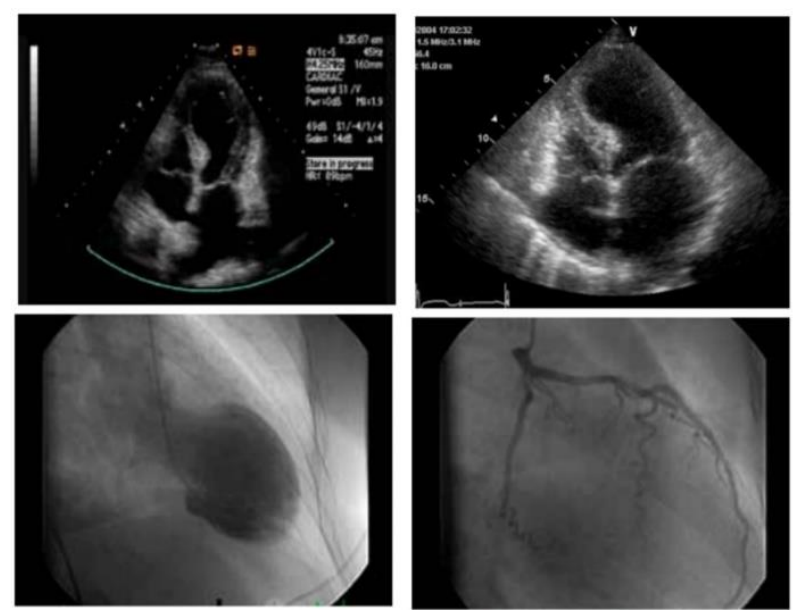

Figure 2: An emergency 2D Echo showing LVEF of $20 \%$ with global hypokinesia, which were suspicious of myocarditis.

Daily investigations: CBC, platelet, creatinine, serum electrolytes, peripheral smear for schistocytes, LDH were sent. Daily PLEX with FFP was done. Daily blood transfusions were given, as per the haemoglobin. Platelet transfusion was done when platelets were less than 10000. The patient did not bleed or require emergency platelet transfusion. As there was no source of active bleed and haemoglobin did not show a rising trend, stool examination was done. Reports showed occult blood, hence, esophagogastroduodenoscopy was done. Day 8: Tracheostomy was done since prolonged ventilation was anticipated. Day 11: T- piece trial was started and was well tolerated. Patient was conscious, and responding well to the verbal commands. Day 13: Vaccination against capsulated organisms was done due to prolonged immunosuppression. Day 16: There was no improvement in the platelet count with course of Rituximab and mycophenolate mofetil, decision to give Eculizumab was taken. Day 17: There was increase in the platelet count, haemoglobin $>8 \mathrm{gm} \%$, LDH showed a falling trend. Decision was taken to do PLEX on alternate days. Day 19: Platelet showed a falling trend, less than $50,000 / \mathrm{mm}^{3}$ and LDH again showed a rising trend. Day 22: Patient and relatives were counselled and explained possibility of resistant HUS-TTP and were offered splenectomy. Patient and attendants refused surgery, so second dose of eculizumab was given. Day 24: Laboratory findings began improving. tracheostomy decannulation trial started and was well tolerated by the patient. Day 28: tracheostomy decannulated. Day 32: Patient was shifted to the ward, speech physiotherapy started. PLEX was being done twice per week. Day 40: Patient underwent last PLEX. Day 43: Patient transferred to another tertiary care hospital due to financial constraints. Day 48: Patient observed a fall in urine output, hence she had increased her fluid intake, after which she developed sudden onset breathlessness. She was taken to the nearby hospital where she was diagnosed to have hypertension with pleural effusion. She was put on non-invasive ventilation, all her labs were within normal limits. Lasix and NTG infusion were started. Day 49: Patient had settled clinically but her blood pressure readings were still on a higher side. Day 52: Oral anti hypertensives and diuretics were started. Patient was shifted on warfarin completely. Currently patient has not had a seizure and she is not on antiepileptic medication currently. Platelet count is stable and her haemoglobin has improved. Her blood pressure showed a sustained increase, hence, she requires antihypertensives and diuretics. Patient is still taking immunosuppressants for APLA and is in regular follow up with a healthy baby.

\section{DISCUSSION}

The classic signs of TTP also known as "Raynaud's Pentad", i.e., haemolytic anaemia, thrombocytopenia, fever, neurologic abnormalities, and renal abnormalities, are seen in only $40 \%$ of patients or in those who present very late or suffer from severe form of the disease. The overall number cases seen have decreased in past few decades as the result of better awareness of the disease. ${ }^{5-6}$ The presence of thrombocytopenia and microangiopathic haemolytic anaemia without other explanation should prompt a suspicion of TTP.

\section{Laboratory testing which is presumptive of TTP should include}

Complete blood count and peripheral blood film for lack of platelets and presence of fragmented red blood cells/schistocytes, serum lactate dehydrogenase, serum creatinine, tests to demonstrate haemolysis like low serum haptoglobin, increased indirect bilirubin, etc, test for coagulation like plasma prothrombin time, bleeding time, clotting time, international normalised ratio. These tests are generally normal unless the patient is in disseminated intravascular coagulation or has severe TTP-HUS, a direct Coombs test to rule out autoimmune haemolytic anaemia, troponin I and electrocardiogram to investigate cardiac involvement, computed tomography/ magnetic resonance imaging of the brain if there are symptoms and signs suggestive of brain injury.

\section{How to proceed with the management?}

In case the suspicion for TTP is high, emergency initiation of therapeutic plasma exchange (TPE) and corticosteroids is considered. Since the disease may present in a way warranting urgent attention, patient 
should be transferred to the centre which can perform TPE, or can be overseen by the clinician who has expertise in it. Many of these patients (>95\%) have plasma ADAMTS13 activity is less than $10 \mathrm{IU} / \mathrm{dl}$ (or $<10 \%$ of normal), and are immune-mediated TTP or iTTP. In these cases, blood sampling for plasma ADAMTS13 testing (e.g.: activity and inhibitors or antiADAMTS13 IgG, etc.) should be done before starting TPE. Daily TPE is initiated as soon as possible with fresh frozen plasma (FFP) or cryo-poor plasma or solvent detergent-treated (SD) plasma as a replacement fluid. The volume of the replacement fluid is 1.0 to 1.5 times of patient's plasma volume (i.e., $40-60 \mathrm{ml} / \mathrm{kg}$ ). Plasma exchange is repeated every 24 hours, until the normalization of patient's platelet counts and serum lactate dehydrogenase. Patients with suspected or confirmed hereditary or congenital TTP (cTTP) are generally treated with plasma infusion (10 to $15 \mathrm{ml} / \mathrm{kg}$ ) at a frequency of every 1 to 3 weeks for maintenance therapy or daily for a symptomatic patient until the symptoms resolve and normalization of platelet counts..$^{7-9}$ As the disease may have a very severe course or cases where the patients present with an acute event, management should be done in a setting with critical/intensive care capabilities, including continuous monitoring of neurologic status, cardiac status, and oxygen saturation. These patients may deteriorate quickly, and are at a high risk of severe organ dysfunction resulting in coma, ischemic stroke, seizures, myocardial infarction, congestive heart failure, arrhythmias, mesenteric ischemia, pancreatitis, and acute kidney injury. Hence early detection, intensive care monitoring, and rapid intervention are needed. ${ }^{10}$

Important points to consider with regard to women's health issues in patients with history of TTP: perinatal care, contraception, pregnancy counselling. The risks of TTP and its unpredictable course must be discussed during the counselling. Pregnancy can trigger TTP relapse, resulting in an increased risk of maternal and foetal morbidity and mortality. It is difficult to predict who may experience a relapse during pregnancy. A normal plasma ADAMTS13 activity at the onset of pregnancy shows a reduced risk of immediate relapse, while a low (Eg. <10 IU/dl) plasma ADAMTS13 activity at the onset of pregnancy may have increased risk of relapse. ${ }^{11}$ In some institutions, women with a decreased ADAMTS13 activity (eg, <10 IU/dl) before or at the onset of pregnancy are offered prophylactic rituximab therapy with a goal to eliminate anti-ADAMTS13 autoantibodies and normalize plasma ADAMTS13 activity before conception. ${ }^{12}$ Patients treated with rituximab are usually asked to wait for 6 to 12 months following rituximab administration before trying to conceive; normalization of CD19 lymphocyte levels and undetectable serum rituximab levels are often used as the evidence of "drug washout." Global drug safety databases suggest that rituximab is associated with few congenital malformations or neonatal infections. ${ }^{13-14}$

\section{What if a female with HUS-TTP becomes pregnant?}

Close monitoring by a haematologist and an obstetrician is required in females with history of TTP with experience in maternal foetal medicine/perinatology. Complete blood counts are usually monitored at least monthly. Plasma ADAMTS13 activity is usually monitored monthly or every 2 to 3 months. More frequent monitoring is required if the ADAMTS13 activity begins to drop during pregnancy. As in the case of TTP in nonpregnant patients, daily TPE is generally needed as soon as possible with FFP, or cryo-poor plasma, or SD plasma as a replacement fluid. Their risk of relapse is generally considered to be high if they are pregnant with plasma ADAMTS13 activity below the normal range. If plasma ADAMTS13 activity falls significantly (eg, usually $<30 \mathrm{IU} / \mathrm{dl}$ or $30 \%$ of normal) even in the absence of clinical signs/symptoms, TPE and corticosteroids (or azathioprine) are often considered. It is a good practice to monitor ADAMTS13 activity throughout pregnancy and during the postpartum period. Induction of labor at 36 to 37 weeks' gestation is commonly suggested. ${ }^{15-16}$ In the absence of other obstetrical indications for cesarean section, vaginal delivery is considered a preferred method of delivery in pregnant women with a history of TTP. This statement also applies to women with a history of either cTTP or iTTP. The uses of antithrombotic therapy in pregnant women with TTP have been controversial. Pregnant women with a history of TTP, and a history of venous thrombosis, are usually offered low molecular weight heparin at prophylactic doses throughout pregnancy, with the goal of preventing the formation of placental microthrombi and insufficiency. Offering antithrombotic therapy to women with a history of TTPassociated pregnancy loss, but not venous thrombosis, also remains controversial.

\section{How about contraceptive counselling?}

The effect of hormonal preparations, particularly those containing estrogen, can trigger a relapse in women with TTP. Women with a history of TTP should be counselled that nonhormonal methods of contraception and progestin-only preparations are preferred over estrogencontaining preparations that may promote produc-ion of autoantibodies against ADAMTS13. ${ }^{17}$

\section{How to tackle with refusal of the blood products?}

Patients with TTP refusing blood products (eg, Jehovah's Witnesses) generally will not accept TPE with replacement of plasma. As this factor is considered by some Clinicians, and patients values are respected, they are counselled to accept albumin and other purified protein fractions because these products are sometimes acceptable. This strategy can, at minimum, help remove ADAMTS13 autoantibodies and other potential harmful inflammatory mediators. Clinicians may empirically consider the use of corticosteroids, rituximab, and caplacizumab as well as erythropoietin and folic acid (to 
promote erythropoiesis). If the patient will accept plasma derivatives, factor VIII concentrates containing sufficient amounts of ADAMTS13 may be considered instead of plasma. If the patient will accept albumin, TPE with albumin as the replacement fluid may be considered.

\section{CONCLUSION}

Pregnancy complicated by HUS- TTP is a rare presentation. It can take a very progressive and a fulminant course. However, an early diagnosis and appropriate treatment is the foremost priority. As in this case, patient had a resistant disease to HUS-TTP, which was precipitated by her pregnancy. Immunomodulators like rituximab, bevacizumab with daily plasma exchange helped this patient in a long run. Patient was on immunosuppressants for a long period of time. But adequate antibiotic coverage was ensured so that she does not go into sepsis.

\section{ACKNOWLEDGEMENTS}

Authors are thankful to Dr. Suvarna khadilkar, head of the department of obstetrics and gynaecology, Bombay hospital, Dr. Shyam Rathi, consultant haematologist, Dr. Sunil Tamvekar, consultant obstetrics and gynaecologist, Dr. Shrirang Bichu, consultant nephrologist, Dr. Farokh Vakil, consultant intensivist, Dr. Nagesh Waghmare, consultant cardiologist, Dr. Vaibhav Somani, consultant gastroenterologist, Dr. Vibhor Pardasani, consultant neurologist and Dr. Sushant Daware, consultant intensivist for their support.

\section{Funding: No funding sources}

Conflict of interest: None declared

Ethical approval: Not required

\section{REFERENCES}

1. Sadler JE, Moake JL, Miyata T, George JN. Recent advances in thrombotic thrombocytopenic purpura. Hematology Am Soc Hematol Educ Program. 2004: 407-23.

2. George JN. How I treat patients with thrombotic thrombocytopenic purpura. Blood. 2010;116(20): 4060-9.

3. Thompson CE, Damon LE, Ries CA, Linker CA. Thrombotic microangiopathies in the 1980s: clinical features, response to treatment, and the impact of the human immunodeficiency virus epidemic. Blood. 1992;80(8):1890-5.

4. Gupta M, Feinberg BB, Burwick RM. Thrombotic microangiopathies of pregnancy: Differential diagnosis. Pregnancy Hypertens. 2018;12:29-34

5. Scully M, Cataland S, Coppo P, de la Rubia J, Friedman KD, Kremer Hovinga J, et al. Consensus on the standardization of terminology in thrombotic thrombocytopenic purpura and related thrombotic microangiopathies. J Thromb Haemost. 2017; 15(2):312-322.

6. Saha M, McDaniel JK, Zheng XL. Thrombotic thrombocytopenic purpura: pathogenesis, diagnosis and potential novel therapeutics. J Thromb Haemost. 2017;15(10):1889-1900.

7. Furlan M, Lämmle B. Aetiology and pathogenesis of thrombotic thrombocytopenic purpura and haemolytic uraemic syndrome: the role of von Willebrand factor-cleaving protease. Best Pract Res Clin Haematol. 2001;14(2):437-54.

8. Kinoshita S, Yoshioka A, Park YD, Ishizashi H, Konno M, Funato $M$, et al. Upshaw-Schulman syndrome revisited: a concept of congenital thrombotic thrombocytopenic purpura. Int $\mathbf{J}$ Hematol. 2001;74(1):101-8.

9. Scully M, Thomas M, Underwood M, Watson $\mathrm{H}$, Langley K, Camilleri RS, et al. Thrombotic thrombocytopenic purpura and pregnancy: presentation, management, and subsequent pregnancy outcomes. Blood. 2014;124(2):211-9.

10. Mörtzell M, Berlin G, Nilsson T, Axelsson CG, Efvergren M, Audzijoni J, et al. Analyses of data of patients with Thrombotic Microangiopathy in the WAA registry. Transfus Apher Sci. 2011;45(2):12531.

11. Ferrari S, Palavra K, Gruber B, Kremer Hovinga JA, Knöbl P, et al. Persistence of circulating ADAMTS13-specific immune complexes in patients with acquired thrombotic thrombocytopenic purpura. Haematologica. 2014;99(4):779-87.

12. Ferrari B, Maino A, Lotta LA, Artoni A, Pontiggia S, Trisolini SM, et al. Pregnancy complications in acquired thrombotic thrombocytopenic purpura: a case-control study. Orphanet J Rare Dis. 2014;9:193.

13. Scully M, Hunt BJ, Benjamin S, Liesner R, Rose P, Peyvandi F, et al. Guidelines on the diagnosis and management of thrombotic thrombocytopenic purpura and other thrombotic microangiopathies. $\mathrm{Br}$ J Haematol. 2012;158(3):323-35.

14. Chakravarty EF, Murray ER, Kelman A, Farmer P. Pregnancy outcomes after maternal exposure to rituximab. Blood. 2011;117(5):1499-506.

15. Pontiggia S, Trisolini SM. Microangiopathic haemolytic anaemia in pregnancy. Transfus Med. Rev. 2018;32:230-6.

16. Watson H, Langley K, Camilleri RS. Current insights into thrombotic microangiopathies: thrombotic thrombocytopenic purpura and pregnancy. Blood. 2015;117(2)530-3.

17. Damon LE, Ries CA, Linker CA. Thrombotic thrombocytopenic purpura associated with oral contraceptives and factor V Leiden: a case report. Cases J. 2009;2:6611.

Cite this article as: Dhungat NP, Kaur S.

Thrombotic thrombocytopenic purpura-hemolytic uremic syndrome in pregnancy in a known case of antiphospholipid antibody syndrome. Int J Reprod Contracept Obstet Gynecol 2022;11:957-61. 